\title{
Acquiring experience in medical humanities teaching: the chicken and egg conundrum
}

\section{B Kirklin}

\section{It is important to try to share with others the understanding gained from reflecting on what we do}

Experience is the child of Thought, and Thought is the child of Action.

We cannot learn men from books.'

S this is the conundrum. How do keen, but inexperienced and under resourced medical educators, follow up their growing enthusiasm for arts and humanities based medical education with high quality effective educational activities? In an ideal world, the encouragement to medical schools from the General Medical Council to incorporate medical humanities into their curriculum $^{2}$ would have been matched with extra funding to develop and deliver these courses, and to provide support for the professional development of those responsible for this important educational input into the training of tomorrow's doctors. The reality is that only three dedicated medical humanities academic posts exist in the UK. The response of educators to this challenge has taken many forms, depending on the local resources and skills available to those involved. In most cases educators are drawing on existing educational and specialist expertise and taking an incremental approach as they incorporate medical humanities methodologies into their work..$^{3-5}$ One proposed way around this obvious gap in experience and specialist expertise within the pool of medical educators is to draw on outside experts. These might come from within affiliated university arts faculties, or be drawn from the growing body of educators who used the literary arts in

Education and debate is edited by Deborah Kirklin. Ideas and items should be sent to her at the address on this page. personal and professional development. ${ }^{6}$ Unfortunately the funds are not always available to allow the latter, and the skills mix in traditional arts faculties does not always match the needs of medical humanities. So for many medical educators, convinced of the benefits that medical humanities might offer their students, the challenge remains of how to acquire experience whilst avoiding treating their students as guinea pigs. The paper by Gull et al that accompanies this editorial provides one model for doing this. The experiences and conclusions of the authors raise a number of interesting points that merit further discussion. As Disraeli stated so eloquently, we gain experience as a consequence of thoughtful reflection on the things we do. The team of educators at Cambridge learnt a number of important things as a result of piloting a special study module in creative writing. They learnt that whether or not it is the intention of the course organisers, creative work like this inevitably draws on the experiences and feelings of the individual learners. They learnt that the boundary between education and therapy is thin in this work and that experience of facilitating educational activities does not necessarily prepare you for this challenge. They also learnt that medical humanities, by taking people out of role can foster mutual respect and improve interdisciplinary working. They learnt that their own role as facilitators is subtle, complex, and sometimes unpredictable.

This group of educators now have an opportunity to revisit the educational objectives for their interesting course and the process through which they hope to achieve these. They have a greater understanding of the power of creative work to enable individuals and groups to improve their personal and professional insights and understanding. Moreover they have a greater understanding of the important and dynamic nature of the group work involved and their key role in facilitating this.

With the establishment, earlier this year, of the Association for Medical Humanities, ${ }^{8}$ and the commitment to hold annual academic conferences, the opportunities for sharing ideas and experiences in medical humanities teaching will increase. To support that process, educators are encouraged to contribute to a national database of medical humanities resources available on line at http://www.mhrd.ucl.ac.uk. The database lists courses offered throughout the UK, provides reviews of teaching resources, and will soon feature online threaded discussions where identifiable, registered users of the site can contribute to debate about issues of relevance to this field.

To misquote Disraeli, we cannot learn medical humanities from books, but we can and should endeavour to share with each other, through publications like this, and community resources like those outlined above, the understanding we gain through reflecting on what we do. In this way we can demonstrate to others the benefits of employing the reflective tools our work encourages others to acquire.

\section{$J$ Med Ethics: Medical Humanities} 2002;28:101-101

Correspondence to: Deborah Kirklin; d.kirklin@pcps.ucl.ac.uk

\section{REFERENCES}

1 Vivian Gray. London: Cassell, 1968: bk one, ch 2 .

2 General Medical Council. Tomorrow's doctors-recommendations on undergraduate medical education. London: General Medical Council, 1993.

3 Kirklin D, Meakin R, Singh S, et al. Living with and dying from cancer: a humanities special study module. Journal of Medical Ethics: Medical Humanities 2000;26:51-4.

4 Glasser B. From Kafka to Casualty: doctors and medicine in popular culture and the arts-a special studies module. Journal of Medical Ethics: Medical Humanities 2001;27:99-101.

5 Evans M, Greaves D. Medical humanities at the University of Swansea. Journal of Medical Ethics: Medical Humanities 2001;27:51-2.

6 Lapidus stands for literary arts in personal development. Find out more at www.lapidus.org.uk

7 Gull SE, O'Flynn R, Hunter JYL. Creative writing workshops for medical education learning from a pilot study with hospital staff. Journal of Medical Ethics: Medical Humanities 2001;28:102-4

8 Association of Medical Humanities. Contact: Dr Robert Arnott; R.G.Arnott@bham.co.u 